Conclusion We have identified and characterized a rare causal variant in MTTP and homozygosity for MTTP p.I564T is associated with progressive NAFLD without any other manifestations of abetalipoproteinemia.

\section{P041 TANDEM MASS TAG-BASED QUANTITATIVE PROTEOMIC PROFILING IDENTIFIES NOVEL PUTATIVE SERUM BIOMARKERS FOR THE DIAGNOSIS OF DRUG-INDUCED LIVER INJURY IN PATIENTS}

\begin{abstract}
${ }^{1}$ Ravi Kodihalli*, 'Vishal Vaidya, 'Zhenyu Wang, 'Joel Federspiel, ${ }^{1}$ Richard Virgen-Slane, ${ }^{1}$ Robert Everley, ${ }^{2,3}$ Jane Grove, ${ }^{2,3}$ Edmond Atallah, ${ }^{4,5}$ Camilla Stephens, ${ }^{4,5} \mathrm{M}$ Isabel Lucena, ${ }^{4,5}$ Raul Andrade, ${ }^{6,7}$ Einar Bjornsson, ${ }^{8}$ Sabine Weber, ${ }^{8}$ Alexander Gerbes, ${ }^{9}$ Guido Stirnimann, ${ }^{10}$ Andrew Fowell, ${ }^{11}$ Hyder Hussaini, ${ }^{1}$ Craig Hyde, ${ }^{12}$ James Dear, ${ }^{2,3}$ Guruprasad Aithal, 'Shashi Ramaiah. 'Worldwide Research Development and Medical, Pfizer, USA; ${ }^{2}$ NIHR Nottingham Biomedical Research Centre, Nottingham University Hospitals NHS Trust and the University of Nottingham, Nottingham, Nottingham, UK; ${ }^{3}$ Nottingham Digestive Diseases Centre, School of Medicine, University of Nottingham, Nottingham, UK; ${ }^{4}$ Servicios de Aparato Digestivo y Farmacologia Clínica, Instituto de Investigación Biomédica de Málaga-IBIMA, Hospital Universitario Virgen de la Victoria, Universidad de Málaga, Malaga, Spain; ${ }^{5}$ Centro de Investigación Biomédica en Red de Enfermedades Hepáticasy Digestivas (CIBERehd), Madrid, Spain; ${ }^{6}$ Department of Gastroenterology, Landspitali University Hospital Reykjavik, University of Iceland, Reykjavik, Iceland; ${ }^{7}$ Faculty of Medicine, University of Iceland, Reykjavik, Iceland; ${ }^{8}$ Department of Medicine II, Klinikum Grosshadern of the University of Munich (KUM), University of Munich, Munich, Germany; ${ }^{9}$ University Clinic for Visceral Surgery and Medicine, University Hospital Inselspital and University of Bern, Bern, Switzerland; ${ }^{10}$ Department of Gastroenterology and Hepatology, Portsmouth Hospitals University NHS Trust, Portsmouth, UK; ${ }^{11}$ Royal Cornwall Hospitals NHS Trust, UK; ${ }^{12}$ Pharmacology, Therapeutics and Toxicology, Centre for Cardiovascular Science, University of Edinburgh, The Queen's Medical Research Institute, Edinburgh, UK
\end{abstract}

\subsection{6/gutjnl-2021-BASL.50}

Background Diagnosis of drug-induced liver injury (DILI) remains a significant challenge in interventional clinical trials during drug development and in clinical practice. We aimed to discover novel diagnostic biomarkers using proteomics and to evaluate their performance characteristics in distinguishing DILI from acute and chronic liver injury from other etiologies.

Methods Adults with idiosyncratic DILI and acute non-DILI liver disease controls were recruited to the Prospective European DILI Registry. Case definitions and causality assessment were performed according to European Association for the Study of the Liver recommendations. Tandem Mass Tag (TMT) labelled quantitative proteomics was performed in serum samples from a discovery set of healthy volunteers (HV, $\mathrm{n}=10$ ), patients with DILI at onset (DO) and recovery (DR) $(\mathrm{n}=10$ each); acute non-DILI controls at onset (NDO) and recovery (NDR) ( $\mathrm{n}=5$ each) and patients with non-alcoholic fatty liver disease $(n=10)$. The performance of top biomarker candidates was assessed in a confirmatory cohort with HV $(n=60)$, DO $(n=83)$, DR $(n=85)$, NDO $(n=35)$ and NDR $(n=21)$ using peptide-based targeted mass spectrometry. Novel biomarkers and patient status were evaluated using univariate and multivariate models.

Results Global proteomic profiling of the discovery cohort revealed 2323 proteins, of which 12 novel biomarkers were chosen based on differential expression, liver specificity, and mechanistic relevance to liver biology/pathogenesis. In the confirmatory analysis, serum concentration of the novel biomarkers was significantly different at DO and returned to levels comparable to $\mathrm{HV}$ during recovery (DR). When the diagnostic performance of these 12 biomarkers was assessed individually, along with the promising biomarker Cytokeratin 18 (CK18, Area Under the Receiver Operator Characteristic (AUROC) Curve $=0.96$ ), the following biomarkers demonstrated the highest sensitivity/specificity (DILI vs HV): Cytoplasmic aconitate hydratase (ACO1, 0.99), Argininosuccinate synthase (ASS1, 0.98), Fumarylacetoacetase (FAH, 0.98), Carbamoyl-phosphate synthase (CPS1, 0.96), Fructose-bisphosphate aldolase B (ALDOB, 0.94), 4-Hydroxyphenylpyruvate dioxygenase (HPD, 0.94), Ornithine carbamoyltransferase (OTC, 0.92).

Conclusion A TMT-based quantitative proteomic profiling approach identified novel putative biomarkers, which are candidates for further evaluation of their role in the diagnosis and prognostication of DILI.

\section{P042 AN AUDIT OF THE KNOWLEDGE OF THE DECOMPENSATED LIVER DISEASE BUNDLE AND ASCITIC TAPS ON PATIENTS PRESENTING ON THE MEDICAL TAKE}

Sabine Bellamy*, Charlotte Ralphs, Jack Stanley, Oliver Arnold, Fiona Gordon. Bristol Royal Infirmary, UK

\subsection{6/gutjnl-2021-BASL.51}

Background The BSG-BASL decompensated cirrhosis care bundle seeks to optimise patient management during the first 24 hours of presentation. The pandemic has impacted on junior doctors' hepatology training, by reducing access to face-to-face teaching. We sought to redress this by assessing how a remote education programme could affect bundle implementation and awareness, shortly after the UK' $s$ first COVID-19 wave.

Methods A retrospective snapshot case-note audit was undertaken for 4 weeks (August 2020) at Bristol Royal Infirmary (BRI). Decompensated cirrhosis admissions were identified from coding and bundle usage and compliance with ascitic tap guidance were quantified. An online survey was conducted of BRI junior doctors (medical) to assess knowledge of the bundle and awareness of appropriate ascitic fluid tests. Subsequent quality improvement interventions were developed, with repeat audits in May-June 2021.

Results The baseline audit identified appropriate bundle completion in $50 \%(\mathrm{n}=8 / 16)$ decompensated cirrhosis patients, none by foundation doctors, with 2 by hepatology consultants. Ascitic tap was attempted in only $50 \%$ of patients with detectable ascites (4/8), with recommended tests requested in 2 of 3 successful taps.

The online survey (40 participants $42 \%$ response) revealed good knowledge of when to use the bundle $(85 \%)$ and how to access it (90\%). For patients with new ascites, $46 \%$ of participants identified appropriate tests, however 39/40 also selected unnecessary options. Knowledge was poorer for patients with known ascites; 20\% selected correct investigations and no participants knew the exact combination to request. Procedural skills varied, 49\% of participants had performed an ascitic tap unsupervised, but 33\% had never attempted one.

After these audits, remote teaching sessions and posters were used to improve prompting of bundle completion; paper copies were distributed to admission areas. Re-audit (May 2020) identified 15 hepatic decompensation admissions, of 